\title{
Fluid modelling of capacitively coupled radio-frequency discharges: a review
}

\author{
L L Alves ${ }^{1}$ and L Marques ${ }^{1,2}$ \\ ${ }^{1}$ Instituto de Plasmas e Fusão Nuclear, Instituto Superior Técnico, Universidade \\ Técnica de Lisboa, 1049-001 Lisboa, Portugal \\ 2 Centro/Departamento de Física da Universidade do Minho, Universidade do \\ Minho, 4710-057, Braga, Portugal \\ E-mail: llalves@ist.utl.pt
}

\begin{abstract}
This paper reviews the basis and the successes with the fluid modelling of capacitively coupled radio-frequency discharges, produced within a parallel-plate cylindrical setup at (single) $13.56-80 \mathrm{MHz}$ frequencies, $6 \times 10^{-2}-6$ Torr pressures, and $50-1000 \mathrm{~V}$ rf-applied voltages, in $\mathrm{SiH}_{4}-\mathrm{H}_{2}, \mathrm{H}_{2}$, and $\mathrm{N}_{2}$. The two-dimensional, time-dependent model used in the simulations, accounts for the production, transport, and destruction of the charged particles (via the electron and ion continuity and momentum-transfer equations, and the electron mean energy transport equations) and of the excited species and/or radicals (via their rate balance equations, including very complete kinetic descriptions with several collisional-radiative production/destruction mechanisms, coupled to the two-term electron Boltzmann equation), accounting also for the self-consistent development of the rf field (via the solution to Poisson's equation). The charged particle transport equations are solved with and without corrective flux terms (due to inertia and friction effects), whose influence in results is discussed. In the case of silane-hydrogen mixtures, the model further includes a phenomenological description of the plasma-substrate interaction to calculate the deposition rate of a-Si:H thin films. In general the model gives good predictions for the self-bias voltage, the coupled power and the intensities of radiative emission transitions (both average and spatially-resolved), underestimating the electron density by a factor of $3-4$.
\end{abstract}

PACS numbers: 52.80.Pi, 52.70.-m, 52.65.Ww, 52.20.-j

Submitted to: Plasma Phys. Control. Fusion 


\section{Introduction}

Capacitively coupled radio-frequency (ccrf) discharges are still among the most powerful and flexible plasma reactors, widely used both in research and in industry. The most popular applications are perhaps those using reactive mixtures of hydrogen with silane or hydrocarbons, to obtain the plasma enhanced chemical vapour deposition (pecvd) of hydrogenated silicon thin films for electronics and photovoltaics $[1,2,3,4]$ or the diamond synthesis $[5,6,7]$, respectively. The content of hydrogen in the mixture provides a straightforward control upon the nature of the films deposited, amorphous or microcrystalline. Nitrogen is another feature gas, due to its high reactivity, and nitrogen-containing rf plasmas are currently used in the processing, modification and funcionalization of different kinds of materials [8, 9, 10, 11, 12, 13, 14]. Nitrogen/methane ccrf dusty plasmas (with $\mathrm{CH}_{4}$ concentrations below 10\%) have also been used in laboratory to study the chemistry of Titan's atmosphere, including the synthesis of analogues of its organic solid aerosols [15]. The obvious interest in these discharges has justified a continuous investment in their study, to understand the phenomena describing their behaviour, to optimize their operation and even to project modified versions of ccrf reactors (e.g. using multi-frequency / multi-excitation approaches). Part of these studies has been driven by the industry, in its demand for higher throughputs, larger processing areas, improved uniformity and film quality, and new opto-electronic properties.

Among the different modelling approaches available to characterize ccrf discharges, two-dimensional fluid models $[16,17,18,19,20,21,22,23,24,25,26,27,28,29,30,31$, 32 ] provide a good compromise solution between calculation runtimes within acceptable values $(<24 \mathrm{hrs}$ ) and (i) the use of a multi-dimensional description, necessary to analyse uniformity issues and to provide a self-consistent electrical description of the discharge, accounting for the driven-to-grounded surface ratio; (ii) a charged particle transport description that captures the essential features associated with the energy transfer to the plasma, the particle distribution within the reactor and the dynamics of the space-charge sheaths; (iii) a high-level of detail for complex chemistries coupled to the base-model, thus allowing a minutia control upon the behaviour of active species controlling the intended application. Reference [33] presents a good review and benchmarking of fluid, particle-in-cell and hybrid models used in the numerical simulation of low-temperature plasma discharges.

For the past 10 years we have been working in the fluid modelling of ccrf discharges in different gases or gas mixtures [27, 28, 29, 30, 32], produced in nondust conditions within reactors similar to the so-called GEC cell $[15,34,35]$, i.e. a parallel-plate cylindrical setup with radius $R=62-69 \mathrm{~mm}$ and interelectrode distance $d=30-40 \mathrm{~mm}$, where the plasma is confined by a grounded lateral grid [see Fig. 1]. This paper reviews the basis and the successes of these studies, highlighting the most impressive results obtained in comparing simulations to measurements. The latter involve (i) electrical diagnostics of the rf applied voltage, the self-bias voltage and 
the effective power coupled to the plasma, (ii) probe or resonant-cavity diagnostics to measure the electron density, and (iii) mass and/or optical emission spectroscopy diagnostics (both average and spatially-resolved) associated to the main excited species of the plasma. More details on these diagnostics can be found in [27, 28, 29, 30, 32].

The two-dimensional, time-dependent model used in the simulations, accounts for the production, transport, and destruction of the charged particles (via the electron and the ion continuity and momentum-transfer equations, and the electron mean energy transport equations) and of the excited species and/or radicals (via their rate balance equations, including very complete kinetic descriptions with several collisional-radiative production/destruction mechanisms, coupled to the two-term electron Boltzmann equation), accounting also for the self-consistent development of the rf field (via the solution to Poisson's equation). These equations are solved subject to appropriate flux conditions at the discharge boundaries. Boundary conditions for the neutral radicals can include a phenomenological description of the plasma-substrate interaction, for example to describe the deposition of thin films. In general the ion transport is described considering some corrective terms in the corresponding momentum-transfer equation, but this correction is usually disregarded in the electron transport equations. Here we present calculation results obtained with and without flux corrections for both the ions and the electrons, because they can eventually modify the current-voltage phase with consequences in the calculated power coupled to the plasma. Simulations cover a wide range of working conditions: rf applied voltages $V_{\mathrm{rf}}=50-1000 \mathrm{~V}$, (single) frequencies $f=13.56-80 \mathrm{MHz}$, and pressures $p=6 \times 10^{-2}-6$ Torr, in $\mathrm{SiH}_{4}-\mathrm{H}_{2}$ mixtures $[27,28]$ and in pure $\mathrm{H}_{2}[29,30]$ and $\mathrm{N}_{2}$ [32]. This paper presents only a fraction of these results, limiting the discussion to changes in the applied voltage and frequency.

\section{Model formulation}

To model ccrf discharges we have coupled a 2D $(r, z)$ time-dependent fluid module (assuming azimuthal symmetry due to the configuration of the rf reactor) to very complete 2D or 0D kinetic modules, for the different gases or gas mixtures considered.

The fluid module solves (i) the continuity and the momentum-transfer equations for charged-particles $\alpha$ ( $\alpha=e, i$ for electrons and ions, respectively)

$$
\begin{aligned}
\frac{\partial n_{\alpha}}{\partial t} & =-\frac{1}{r} \frac{\partial\left(r \Gamma_{\alpha_{r}}\right)}{\partial r}-\frac{\partial \Gamma_{\alpha_{z}}}{\partial z}+S_{\alpha} \\
\Gamma_{e_{q}} & =-\left(\mu_{e} N\right) n_{e} \frac{E_{q}}{N}-\frac{1}{N} \frac{\partial\left[\left(D_{e} N\right) n_{e}\right]}{\partial q}+\Gamma_{\operatorname{corr}_{q}} \\
\Gamma_{i_{q}} & = \pm\left(\mu_{i} N\right) n_{i} \frac{E_{i_{q}}^{\mathrm{eff}}}{N}-\frac{1}{N} \frac{\partial\left[\left(D_{i} N\right) n_{i}\right]}{\partial q}( \pm \text { for }+/- \text { ions }) ;
\end{aligned}
$$

(ii) the electron mean energy transport equations

$$
\frac{\partial\left(n_{e} \varepsilon\right)}{\partial t}=-\frac{1}{r} \frac{\partial\left(r \Gamma_{\varepsilon_{r}}\right)}{\partial r}-\frac{\partial \Gamma_{\varepsilon_{z}}}{\partial z}-\vec{\Gamma}_{e} \cdot \vec{E}-S_{\varepsilon}
$$




$$
\Gamma_{\varepsilon_{q}} \quad=-\left(\mu_{\varepsilon} N\right) \varepsilon n_{e} \frac{E_{q}}{N}-\frac{1}{N} \frac{\partial\left[\left(D_{\varepsilon} N\right) \varepsilon n_{e}\right]}{\partial q}
$$

and (iii) Poisson's equation

$$
\frac{1}{r} \frac{\partial}{\partial r}\left(r \frac{\partial V}{\partial r}\right)+\frac{\partial^{2} V}{\partial z^{2}}=-\frac{e}{\varepsilon_{0}}\left(\sum_{i} n_{i}-n_{e}\right) .
$$

Here, $n_{\alpha}$ is the particle density; $\Gamma_{\alpha_{q}}$ are the $q=r, z$ components of the particle flux; $S_{\alpha}$ is the net production rate of particles due to kinetic mechanisms; $N=p / k_{B} T_{g}$ is the gas density ( $k_{B}$ is the Boltzmann's constant and $T_{g}$ is the gas temperature); $\mu_{\alpha} N$ and $D_{\alpha} N$ are the reduced mobility and diffusion coefficient, respectively; $E_{i_{q}}^{\text {eff }} / N$ is the $q$ component of the so-called reduced effective electric field [28]; $\varepsilon$ is the electron mean energy; $\Gamma_{\varepsilon_{q}}$ is the $q$ component of the electron energy flux; $\mu_{\varepsilon} N$ and $D_{\varepsilon} N$ are the reduced mobility and diffusion coefficient for energy transport, respectively; $S_{\varepsilon}$ is the net power density lost by the electrons due to (elastic and inelastic) collisions; $V$ is the rf potential which relates to the rf electric field through $\vec{E}=-\vec{\nabla}_{r, z} V ; e$ and $\varepsilon_{0}$ are the electron charge and the vacuum permittivity, respectively; and $\Gamma_{\operatorname{corr}_{q}}$ is a correction to the drift-diffusion approximation with the electron particle flux.

The reduced effective electric field (for the ions) is defined using [28]

$$
\frac{\partial\left(E_{i_{q}}^{\mathrm{eff}} / N\right)}{\partial t}=-\frac{1}{\mu_{i} N}\left(v_{i_{r}} \frac{\partial}{\partial r}+v_{i_{z}} \frac{\partial}{\partial z}\right) v_{i_{q}}-\frac{\nu_{i}}{\mu_{i} N} v_{i_{q}}+\nu_{i}^{c}\left(\frac{E_{q}}{N}-\frac{E_{i_{q}}^{\mathrm{eff}}}{N}\right),
$$

and the flux correction (for the electrons) writes

$$
\Gamma_{\text {corr }_{q}}=-\frac{n_{e}}{\nu_{e}^{c}}\left[\frac{\partial v_{e_{q}}}{\partial t}+\left(v_{e_{r}} \frac{\partial}{\partial r}+v_{e_{z}} \frac{\partial}{\partial z}\right) v_{e_{q}}+\nu_{e} v_{e_{q}}\right],
$$

where $v_{\alpha_{q}} \equiv \Gamma_{\alpha_{q}} / n_{\alpha}$ is the $q$ component of the $\alpha$-particle drift velocity, $\nu_{\alpha} \equiv S_{\alpha} / n_{\alpha}$ is the $\alpha$-particle net production frequency, and $\nu_{\alpha}^{c}$ is the electron/ion-neutral momentumtransfer collision frequency. The latter relates to the particle mobility, according to $\mu_{\alpha} N=e /\left[m_{\alpha}\left(\nu_{\alpha}^{c} / N\right)\right]$ with $m_{\alpha}$ the $\alpha$-particle mass. In many works calculations neglect: (i) for the ions, the first two terms on the right-hand side of equation (4), describing non-linear inertia effects and additional friction due to collisions, respectively; (ii) for the electrons, the whole corrections $\Gamma_{\operatorname{corr}_{q}}$ with equation (5), whose terms on the righthand side account for the time-variation of the velocity, the inertia and the friction, respectively. Here we will test, for both electrons and ions, the influence on results of these flux corrections.

Equations (1a)-(3) are solved within the plasma reactor (corresponding to a $2 \mathrm{D}$ workspace delimited by the discharge axis at $r=0$, the grounded lateral grid at $r=R$, the driven electrode at $z=0$, and the grounded electrode at $z=d$, see Fig. 1), subject to the following boundary conditions: (i) symmetry boundary conditions, at the reactor axis, for scalar quantities (densities, mean energy, potential); (ii) the value of the rf potential at each physical boundary: 0 at the grounded electrode and walls and $V_{\mathrm{dc}}+V_{\mathrm{rf}} \cos (2 \pi f t)$ at the driven electrode, where $V_{\mathrm{dc}}$ is the self-bias voltage, an offset potential self-consistently calculated within the model, which develops in the case 
of an asymmetric reactor (with more surfaces grounded than driven); (iii) conditions for the perpendicular component of the fluxes $(\perp)$ at the different electrodes and walls, assumed as totally absorbing boundaries $[28,36]\left(\Gamma_{e}\right)_{\perp}=(1 / 2) n_{e}\langle v\rangle-\xi_{e} \sum_{i}\left(\Gamma_{i}\right)_{\perp}$; $\left(\Gamma_{\varepsilon}\right)_{\perp}=(1 / 2) n_{e}\langle v u\rangle-\xi_{e} \varepsilon \sum_{i}\left(\Gamma_{i}\right)_{\perp} ;\left(\Gamma_{i}\right)_{\perp}=(1 / 4) n_{i} v_{\mathrm{th}_{i}}+\beta_{i} n_{i}\left(\mu_{i} N\right)\left(E_{i_{\perp}}^{\mathrm{eff}} / N\right)$, where $\langle$.$\rangle represents an average value over the electron energy distribution function (eedf) and$ $u \equiv m_{e} v^{2} /(2 e)$ is the electron kinetic energy (in $\left.\mathrm{eV}\right), \xi_{e}$ is the secondary electron emission coefficient (here, we assume that secondary emission occurs at the driven electrode only, with $\left.\xi_{e}=0-1\right), v_{\mathrm{th}_{i}}=\left(8 k_{B} T_{g} / \pi m_{i}\right)^{1 / 2}$ is the thermal velocity for the $i$-ion species, and $\beta_{i}=1,0$ for $E_{i_{\perp}}^{\text {eff }}$ directed towards the wall and in the opposite direction to the wall, respectively.

The kinetic module solves the stationary rate balance equations for the different neutral (molecular and atomic) species $k$ considered [27],

$$
\frac{1}{r} \frac{\partial\left(r \Gamma_{k_{r}}\right)}{\partial r}+\frac{\partial \Gamma_{k_{z}}}{\partial z}=S_{k}
$$

where $\Gamma_{k_{q}}=-D_{k}\left(\partial n_{k} / \partial q\right)$ and $n_{k}$ are the species flux $q$-component and density, respectively, $D_{k}$ is the diffusion coefficient, and $S_{k}$ is a source term accounting for the net production of $k$-species. The use of a stationary approach is justified considering the long evolution times ( $>0.1 \mathrm{~ms}$ ) of the heavy species, compared to $\mathrm{rf}$ periods of $\sim 10-80$ ns. Moreover, convection fluxes can be neglected when compared to diffusion fluxes (Peclet's numbers $P e \ll 1$ ). The flux boundary conditions to equation (6) can be used to describe plasma-surface interactions, following a phenomenological approach. In the case of solid surfaces (electrodes, walls) the boundary fluxes are set to satisfy the Milne's condition $[37,38]$

$$
\left.\Gamma_{k_{q}}\right|_{\text {wall }}=-\left.D_{k} \frac{\partial n_{k}}{\partial q}\right|_{\text {wall }}=\left.\frac{\zeta_{k}}{1-\zeta_{k} / 2} \frac{v_{\mathrm{th}_{k}}}{4} n_{k}\right|_{\text {wall }} \quad,
$$

where $\zeta_{k} \equiv s_{k}+\gamma_{k}$ is the total wall loss probability for the $k$-species, with $s_{k}$ the sticking probability (representing the contribution of a radical to deposition) and $\gamma_{k}$ the recombination probability (representing the probability of reaction between the $k$ species and another adsorbed species, to form a stable product that returns to the plasma volume). In the case of a partially transparent surface (grid), with an opacity coefficient $A$, we adopt a modified form of equation (7) [39] to write the fluxes at each side of the surface (designated by $i, j=1,2$ )

$$
\left.\Gamma_{k_{q_{i}}}\right|_{\text {grid }}=-\left.l_{k} \frac{\partial n_{k_{i}}}{\partial q}\right|_{\text {grid }}=(-1)^{i}\left[a_{k} n_{k_{i}}-n_{k_{j}}\right] \text {, }
$$

where $l_{k} \equiv\left(D_{k} / v_{\mathrm{th}_{k}}\right) A\left(2-A+2 r_{k}+r_{k}^{2} A\right) /(1-A)$ and $a_{k} \equiv 1+A^{2}\left(1-r_{k}^{2}\right) /[2(1-A)]$, with $r_{k} \equiv 1-\zeta_{k}$ the reflexion coefficient for the $k$-species. Often the kinetic module solves only the space-average version of equations (6), to limit the calculation runtime due to the high number of species and kinetic processes considered, in which case we impose the density profiles of the different neutral species [30].

The rate balance equations (6) are solved coupled to the two-term homogeneous and stationary electron Boltzmann equation, accounting for inelastic and superelastic 
collisions. This yields the eedf and the electron parameters (transport and rate coefficients) with equations $(1 a)-(2 b),(4)-(5)$ and the boundary conditions, which are calculated as integrals of cross sections over the eedf [36]. The space-time dependence of these parameters is defined by adopting the local mean energy approximation [28, 36], i.e. assuming that the space-time variation of the eedf, $f(u, r, z, t)$, is introduced via the local electron mean energy profile $f(u, r, z, t)=f(u)_{\mid \varepsilon(r, z, t)}$. The step-by-step procedure is the following: first, the homogeneous and stationary eedf, $f(u)$, is calculated from the kinetic module at various $E / N$, given the composition of the gas/plasma system (including the vibrational distribution function); second, the eedfs obtained are used to calculate the different electron parameters, which are then tabulated as a function of $\varepsilon_{\text {eedf }}=\int_{0}^{\infty} u^{3 / 2} f(u) d u$; third, the local values of the electron mean energy $\varepsilon(r, z, t)$, obtained from the solution to the electron energy balance equation $(2 a)$, are used in the table constructed to define the space-time map of the electron transport and rate coefficients. Note that other authors [40, 41, 42] have preferred to adopt a time-consuming hybrid approach, calculating the space-time profiles of the electron parameters by using a multidimensional, time-dependent Monte-Carlo code coupled to the fluid equations.

More details about the model equations and formulation, the procedure followed in the numerical solution, and the kinetic data adopted (collisional cross sections, rate coefficients, and transport parameters) for the different gases can be found in $[27,28,29,30,32]$.

\section{Results}

\subsection{Silane-hydrogen mixtures}

Hydrogenated amorphous silicon a-Si:H thin films, extensively used in microelectronics, can be obtained by pecvd from a precursor mixture of $\mathrm{SiH}_{4}-\mathrm{H}_{2}$. Our study of these discharges focused on the deposition rate $V_{d}$ of these films, a key parameter to control in applications, aiming to answer the following questions: (i) is it possible to predict $V_{d}$ using a simple phenomenological approach?; (ii) what radicals / ions contribute the most for these depositions?

Figure 2 presents the time-average axial profiles of the charged particles, plotting the total densities of electrons and ions in Fig. 2(a) (where one can observe the charge separation within the boundary sheaths) and discriminating the densities of the most important ions with the $\mathrm{SiH}_{4}-\mathrm{H}_{2}$ plasma in Figs. 2(b)-(c) (where one can conclude that $\mathrm{SiH}_{3}^{+}$and $\mathrm{H}_{3}^{+}$are the dominant ion species, even at low concentrations of silane). In general the charged particles are produced in the sheath regions, although their maximum density is found at the discharge centre due to transport effects [see Fig. 2(a)]. Note the difference between the profiles of $\mathrm{H}_{2}^{+}$and of $\mathrm{H}^{+}$and $\mathrm{H}_{3}^{+}$, the former being converted into $\mathrm{H}_{3}^{+}$just after its formation, which contributes to preserve the maxima of the $\mathrm{H}_{2}^{+}$density profile in the positions where this species is originally produced. 
Figure 3 shows simulations and measurements of the time-average axial profiles of the $\mathrm{SiH}$ and $\mathrm{SiH}_{2}$ radical densities, obtained for different work conditions. These results are an upgrade of those reported in [27], involving the validation of the model and the improvement of its predictions. The main changes are: (i) the rate coefficient for the reaction $\mathrm{SiH}_{2}+\mathrm{SiH}_{4} \longrightarrow \mathrm{Si}_{2} \mathrm{H}_{6}$ now reads $2.2 \times 10^{-10}\left[1-(1+1.72 p)^{-1}\right] \mathrm{cm}^{3} \mathrm{~s}^{-1}$ [44], where $p$ is in Torr; (ii) we have considered the loss channels for dimmers listed in Table 1 [43]; (iii) the $\mathrm{SiH}_{2}$ wall loss probability was decreased to 0.6 [44]. The results show that the kinetic model for silane needs further adjustments to improve radical density predictions.

Table 2 summarizes the loss probabilities of radicals adopted in the model, which were used to calculate the deposition rate of a-Si:H thin films. This quantity is written as a balance between the adsorption flux $\Phi_{j}$ of Si-containing radicals/ions $j$ and the etching flux $\Phi_{i_{H}}$ of hydrogen atoms/ions $i_{H}$

$$
V_{d}=\frac{1}{n_{s}}\left(\sum_{j} S_{j} \Phi_{j}-\sum_{i_{H}} S_{i_{H}} \Phi_{i_{H}}\right),
$$

where $n_{s}$ is the surface density of the a-Si:H film, $S_{j}$ and $S_{i_{H}}$ are stoichiometry factors, and $\Phi_{j}=n_{j}\left(v_{\mathrm{th}_{j}} / 4\right) \zeta_{j}\left(1-\zeta_{j} / 2\right)^{-1}\left(s_{j} / \zeta_{j}\right)$ (a similar expression writes for $\Phi_{i_{H}}$ ), with $n_{j}$ the density of radical $j$. Model predictions for the radial profile of $V_{d}$ are depicted in Fig. 4, where one can observe a good agreement with experimental values measured by profilometry. The contribution of ions (mainly $\mathrm{SiH}_{3}^{+}$and $\mathrm{SiH}_{2}^{+}$) for the deposition is typically of $9 \%$ for silane dominated conditions, in which case the thin film formation is controlled in equal parts by the monomers $\mathrm{SiH}_{3}$ and $\mathrm{SiH}_{2}$, the dimmer radicals having a minor participation only.

\subsection{Hydrogen}

Hydrogenated microcrystalline silicon $\mu \mathrm{c}-\mathrm{Si}: \mathrm{H}$ thin films, used in solar cells production, can be obtained from $\mathrm{SiH}_{4}-\mathrm{H}_{2}$ mixtures under high dilution conditions for silane. This was the main motivation driving our study of pure hydrogen plasmas, where we have used frequencies above the standard $13.56 \mathrm{MHz}$ to answer the following questions: (i) how does the power coupled to the plasma depends on the working conditions?; (ii) does an increase in the frequency lead to an enhancement in the population of H-atoms, thus favouring the fast deposition of high-quality films?

Figure 5 presents, as a function of $V_{\text {rf }}$ for various frequencies, the power coupled to the plasma $W_{\text {eff }}$, the time-average plasma potential at discharge centre $V_{p}(0, d / 2)$, and the self-bias voltage, calculated and measured for pure $\mathrm{H}_{2}$ discharges. The quantity power coupled is defined as $W_{\text {eff }}=f \int_{0}^{(1 / f)}\left[V_{\mathrm{dc}}+V_{\mathrm{rf}} \cos (2 \pi f t)\right] I_{\mathrm{rf}}(t) d t$, where $I_{\mathrm{rf}}$ is the total rf-current (conduction and displacement) collected at the driven electrode. As anticipated, the coupled power increases with both $V_{\text {rf }}$ and $f$. One observes a good agreement between simulations of measurements of $W_{\text {eff }}$ and $V_{p}$ (this behaviour extends to frequencies up to $80 \mathrm{MHz}$ and to other pressures) and an underestimation of the calculated $\left|V_{\mathrm{dc}}\right|$ with respect to experiment. The calculations were done including the 
corrective terms in the ion flux equation $(1 c)$. The impact on results of including the electron flux corrective terms is minimal [cf. short-dotted curves in Figs. 5(a), (c) and Fig. 6(a)], whereas removing the corrective terms in the ion flux equation leads to an increase of $21 \%$ in $W_{\text {eff }}$ and a decrease of $5 \%$ and $9 \%$ in $\left|V_{\mathrm{dc}}\right|$ and $n_{e}$ respectively, at $V_{\text {rf }}=300 \mathrm{~V}, f=13.56 \mathrm{MHz}$ and $p=0.3$ Torr (these corrections are higher at high $V_{\mathrm{rf}}$ and $f$, and at low $p$ ).

Figure 6 plots the time-average densities of the electrons and the $\mathrm{H}$ atom (at given positions in the discharge), as a function of $V_{\mathrm{rf}}$ for various frequencies. As usually observed in the fluid modelling of ccrf discharges, simulations underestimate the measured values of $n_{e}$ by a factor of $3-4$, with impact on the densities of all species (such as $\mathrm{H}$ ) that depend on the electron kinetics. However, the model predicts a correct qualitative variation for $n_{e}$ and $n_{\mathrm{H}}$ with $V_{\mathrm{rf}}$ and $f$ (and also with $p$ ), only possible due to the use of in-situ measurements for the wall recombination probability of $\mathrm{H}$ [30], confirming an increase in the population of H-atoms with the rf frequency.

The disagreement between calculated and measured values of $V_{\mathrm{dc}}$ indicates that model predictions overestimate the ion flux towards the boundaries. This is probably associated with an overestimation of the space-charge field intensity at the reactor walls, hence an extraordinary energy consumption for its maintenance. Ultimately this can be responsible for limiting the energy available for electron production, justifying the low electron density values obtained in the simulations.

\subsection{Nitrogen}

Radio-frequency discharge plasmas produced in nitrogen and nitrogen mixtures are becoming increasingly popular, because they exhibit a high chemical reactivity leading to the production of active radicals and ions, even at low pressure and ambient temperature. We have engaged a step-by-step methodology to study nitrogen-containing ccrf plasmas (e.g. produced using $\mathrm{N}_{2}-\mathrm{H}_{2}$ and $\mathrm{N}_{2}-\mathrm{CH}_{4}$ mixtures), which started with the modelling of pure $\mathrm{N}_{2}$ plasmas addressing the following basic questions: (i) is it possible to describe the main kinetic features with these plasmas, by coupling our fluid model to a very complete collisional-radiative model for nitrogen?; (ii) is it reasonable to use such a highly-complex scheme, considering the uncertainties associated with some of the collisional data required?

Figure 7 presents the axial profile of the radiative intensity with the $\mathrm{N}_{2} \operatorname{SPS}(0,0)$ band, measured (along the discharge cross section) and calculated (applying a timeand radial- average), whereas Fig. 8 plots, as a function of $W_{\text {eff }}$, the space-time average radiative intensities of the $\operatorname{SPS}(0,2)$ and the $\operatorname{FNS}(0,0)$ bands with $\mathrm{N}_{2}$, obtained at various pressures. These figures contribute to validate the transport-kinetic features described by the 2D fluid model, whose predictions for the nitrogen radiative intensities are in reasonable good agreement with both spatially-resolved and spatially-averaged spectroscopy measurements. Notice that this experiment is difficult to control, not only due to the extreme dependence of the emitted light intensity with position but also 
because of the limitations in defining a precise reference frame between the electrodes. Notice further that, at low pressures, the inclusion in equation (4) of the non-linear inertia terms for $\mathrm{N}_{4}^{+}$introduces numerical instabilities in model convergence.

The kinetic scheme adopted (for $\mathrm{N}_{2}$ and the other gases) depends on several parameters (branching ratios, secondary emission coefficient, wall loss probabilities), whose high uncertainty can influence the model results. In the particular case of $\mathrm{N}_{2}$ discharges, simulations tests show that: (i) the branching ratios for the ion and the atom production can vary between $0-1$ with little influence upon the results; (ii) variations, between 0 and 0.5 , in the secondary electron emission coefficient produce negligible changes in the electron density, reducing $\left|V_{\mathrm{dc}}\right|$ by $\sim 10 \%$ at 0.38 Torr and $10 \mathrm{~W}$; (iii) the wall atomic recombination probability has a direct effect upon the dissociation degree, which decreases by a factor of $\sim 10^{2}$ (leading to an increase of $\sim 12 \%$ in $n_{e}$ ) when that probability varies between $10^{-3}$ and 1 ; (iv) an increase in the wall vibrational deeexcitation probability from $4.5 \times 10^{-4}$ to 1 at 0.38 Torr and $10 \mathrm{~W}$ decreases the dissociation degree by $\sim 93 \%$ and increases $n_{e}$ by $\sim 45 \%$. Notice that even if these probabilities are set to unity the dissociation degree remains low $(<4 \%)$ and $n_{e}$ increases not more than $45 \%$. This shows that the highly-complex kinetic scheme proposed here is able to capture the main plasma features (within experimental errors), even considering the uncertainties associated with some of the collisional data.

\section{Final remarks}

The 2D time-dependent fluid modelling of ccrf plasma reactors gives a correct overall description of their main operation features, over a wide range of operation conditions: rf voltages, frequencies, pressures and mixture compositions. The main simulation results are (i) electrical quantities, such as the plasma potential, the self-bias voltage and the effective electrical power coupled to the plasma, and (ii) the densities and fluxes of the different plasma species (neutral and charged), from which one can deduce the intensities of radiative emission transitions and the deposition/etching rates (if the model includes some description of the plasma-substrate interaction). Modelling results are meaningful only if validated by comparison with experimental values, hence the research study justifying the present paper.

In general, the deviations of model predictions with respect to measurements are mainly related to uncertainties in the volume and surface kinetic data and to limitations in the description of boundary phenomena. Changes in the rate coefficients and/or the reactions with the volume and the surface kinetics have a direct impact on results, thus justifying a continuous investment in the definition of the collisional-radiative mechanisms controlling these gas discharges. Corrections depend on the specific system under analysis, as discussed here in the calculation of the silane radical populations, in the estimation of the hydrogen and nitrogen density atoms, and in the analysis of the charged particle flux corrections, whose impact on results is negligible for hydrogen and may be relevant for nitrogen. 
Fluid models are powerful tools for the modelling of ccrf discharges, because they are able to provide an adequate description of the complex physical/chemical mechanisms relevant for many processing applications. However they have some limitations not fully addressed yet. More work is needed to correctly estimate the space-time dependence with the electron transport parameters, whose modification can lead to considerable changes in both the particle and the energy balance. Note that the validity of the local mean energy approximation has never been theoretically demonstrated in the low-pressure region adopted here. Even with these limitations, fluid simulations provide a good compromise solution between fast calculations and a detailed physical characterization of ccrf discharges.

\section{Acknowledgments}

Work partially supported by the FCT under the Pest-OE/SADG/LA0010/2011 and the Pest-C-FIS/UI607/2011 projects. 
Figure captions

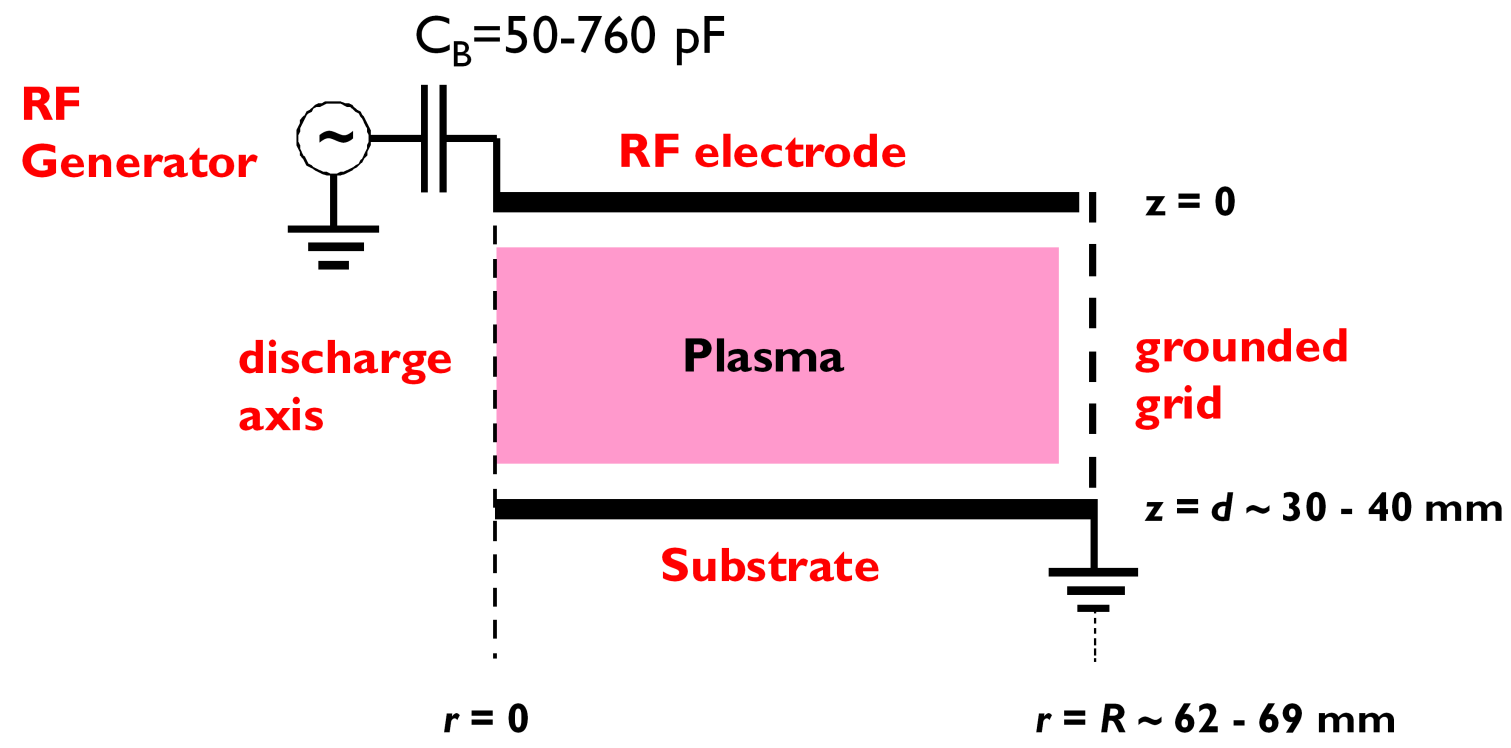

Figure 1. Schematic diagram of the plasma chamber with the capacitively coupled reactors used in this work. $C_{B}$ represents the blocking capacitor that imposes $V_{\mathrm{dc}}$. 

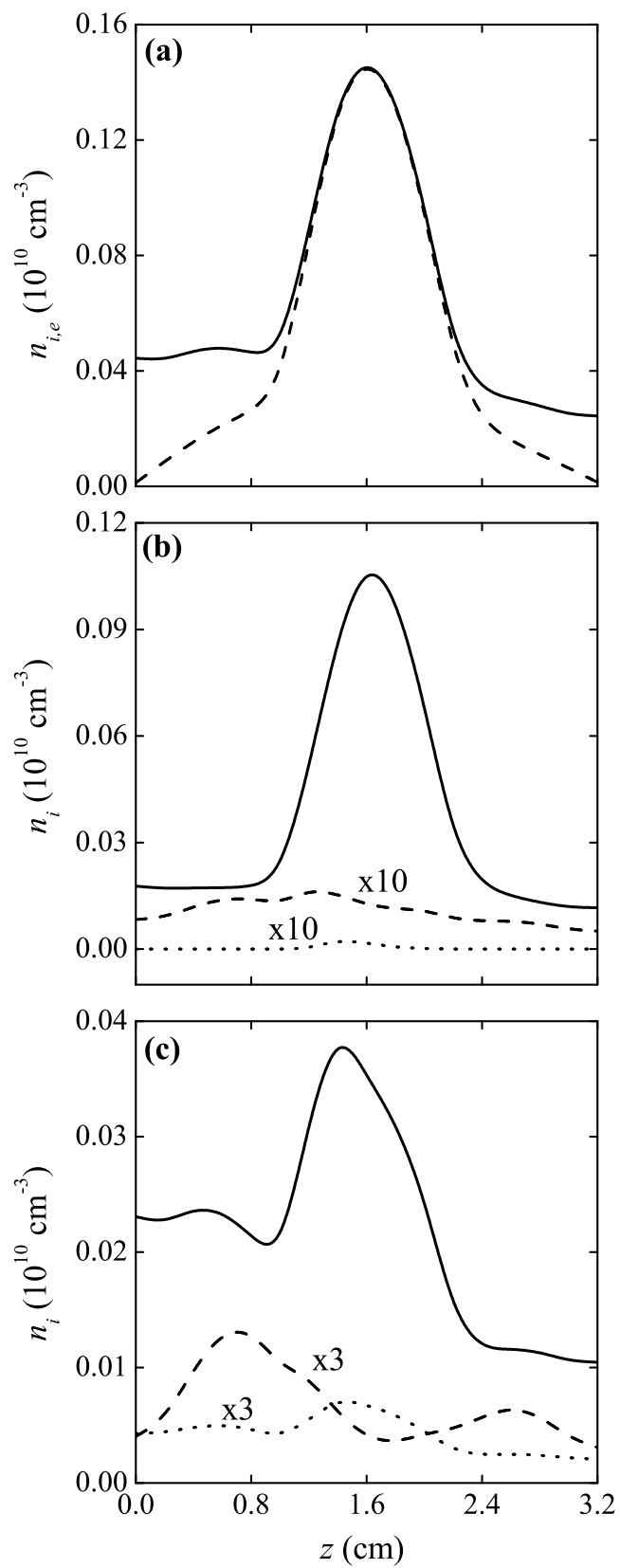

Figure 2. Time-average axial profiles of the charged particle densities at $r=0$, for a $7 \%-\mathrm{SiH}_{4}: 93 \%-\mathrm{H}_{2}$ discharge at $V_{\mathrm{rf}}=450 \mathrm{~V}$ and $p=0.3$ Torr. (a) Total ion density (solid curve), electron density (dashed). (b) Silane ion densities: $\mathrm{SiH}_{3}^{+}$(solid), $\mathrm{SiH}_{2}^{+}$(dashed), $\mathrm{SiH}_{3}^{-}$(dotted). The $\mathrm{SiH}_{2}^{+}$and $\mathrm{SiH}_{3}^{-}$densities are multiplied by 10 . (c) Hydrogen ion densities: $\mathrm{H}_{3}^{+}$(solid), $\mathrm{H}_{2}^{+}$(dashed), $\mathrm{H}^{+}$(dotted). The $\mathrm{H}_{2}^{+}$and $\mathrm{H}^{+}$ densities are multiplied by 3 . 

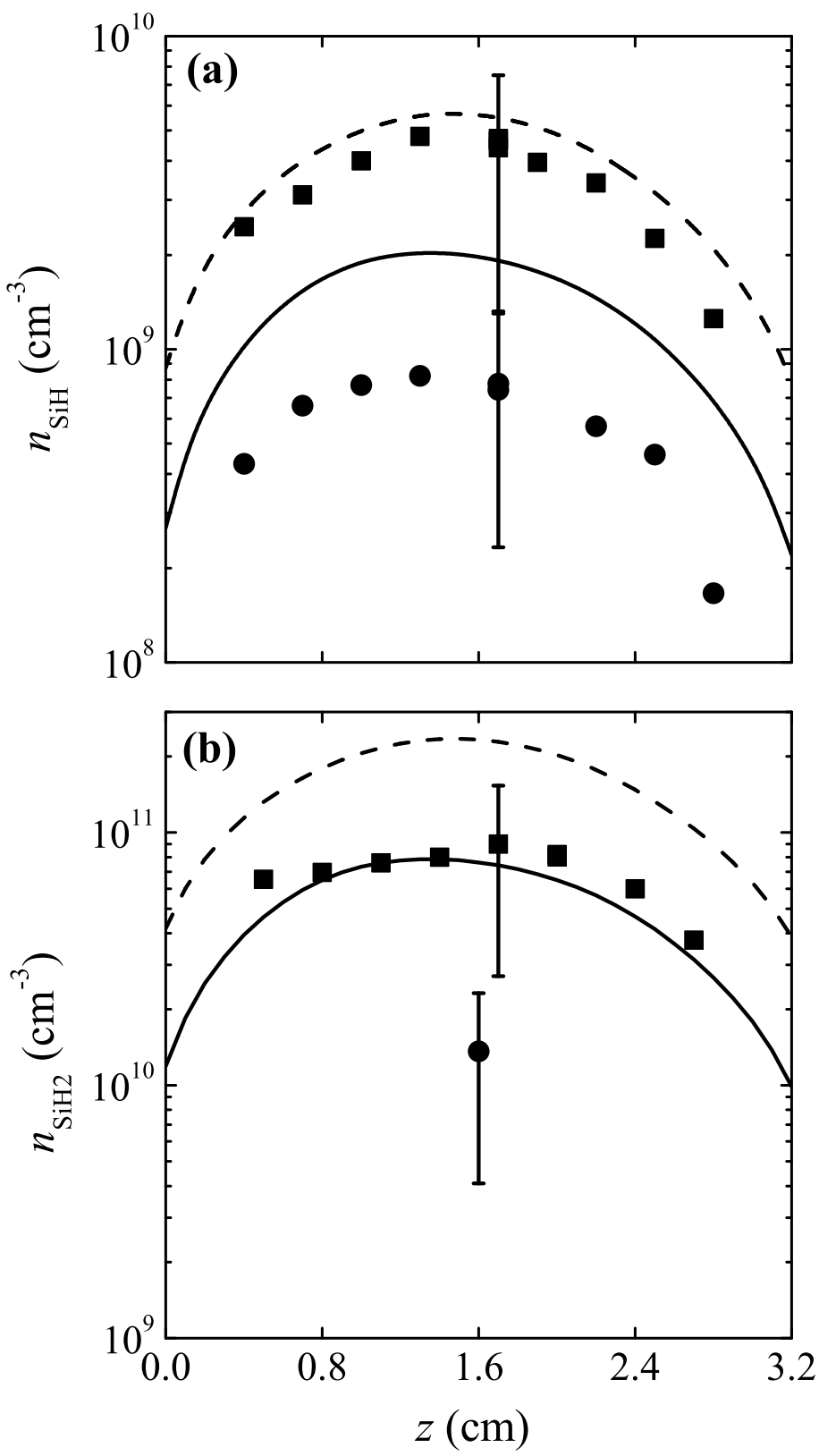

Figure 3. Time-average axial profiles of the $\mathrm{SiH}(\mathbf{a})$ and $\mathrm{SiH}_{2}$ (b) densities, calculated (curves) and measured by LIF (points) in the following conditions: $83 \%-\mathrm{SiH}_{4}: 17 \%-\mathrm{H}_{2}$ at $V_{\mathrm{rf}}=160 \mathrm{~V}$ and $p=68 \mathrm{~m}$ Torr (solid curves and circles); $60 \%-\mathrm{SiH}_{4}: 40 \%-\mathrm{H}_{2}$ at $V_{\mathrm{rf}}=490 \mathrm{~V}$ and $p=68 \mathrm{mTorr}$ (dashed and squares). 


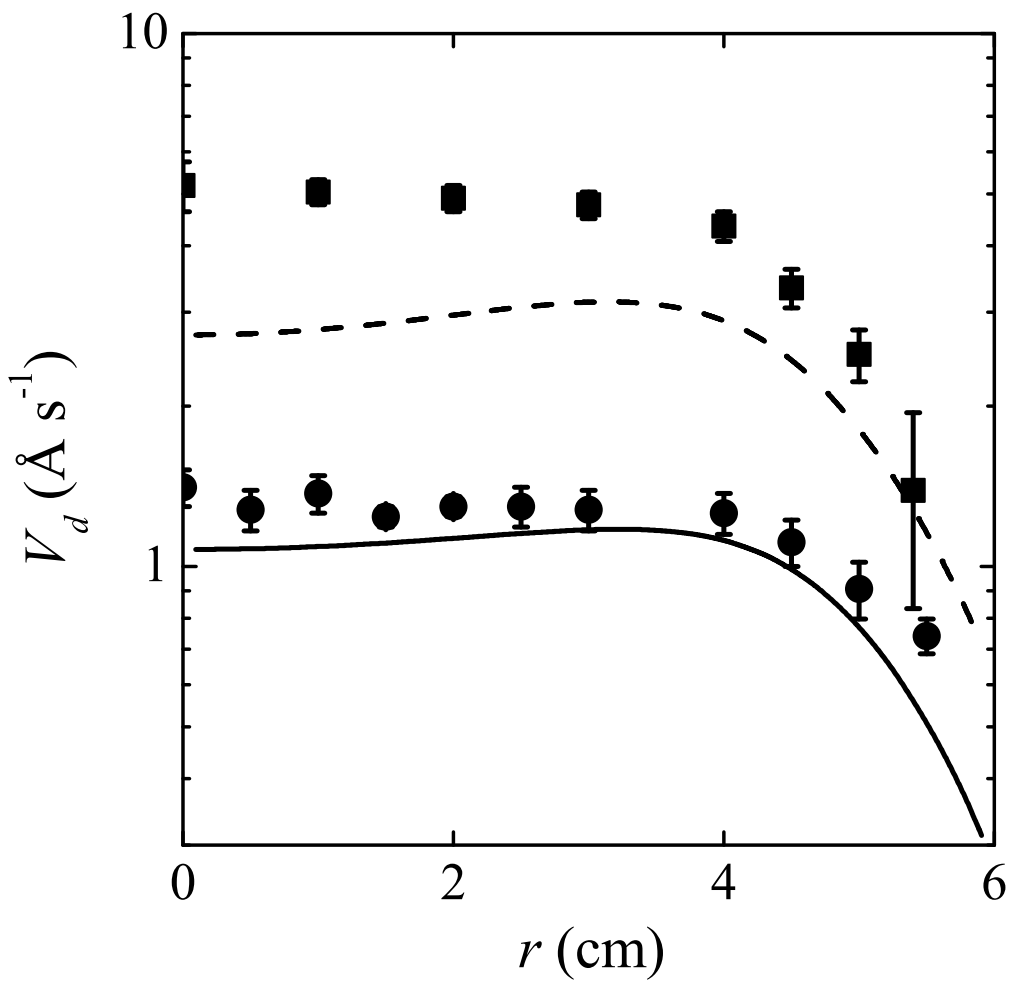

Figure 4. Deposition rate of the a-Si:H thin film, along the radius of the substrate, calculated (curves) and measured by profilometry (points) in the same conditions of Fig. 3. 

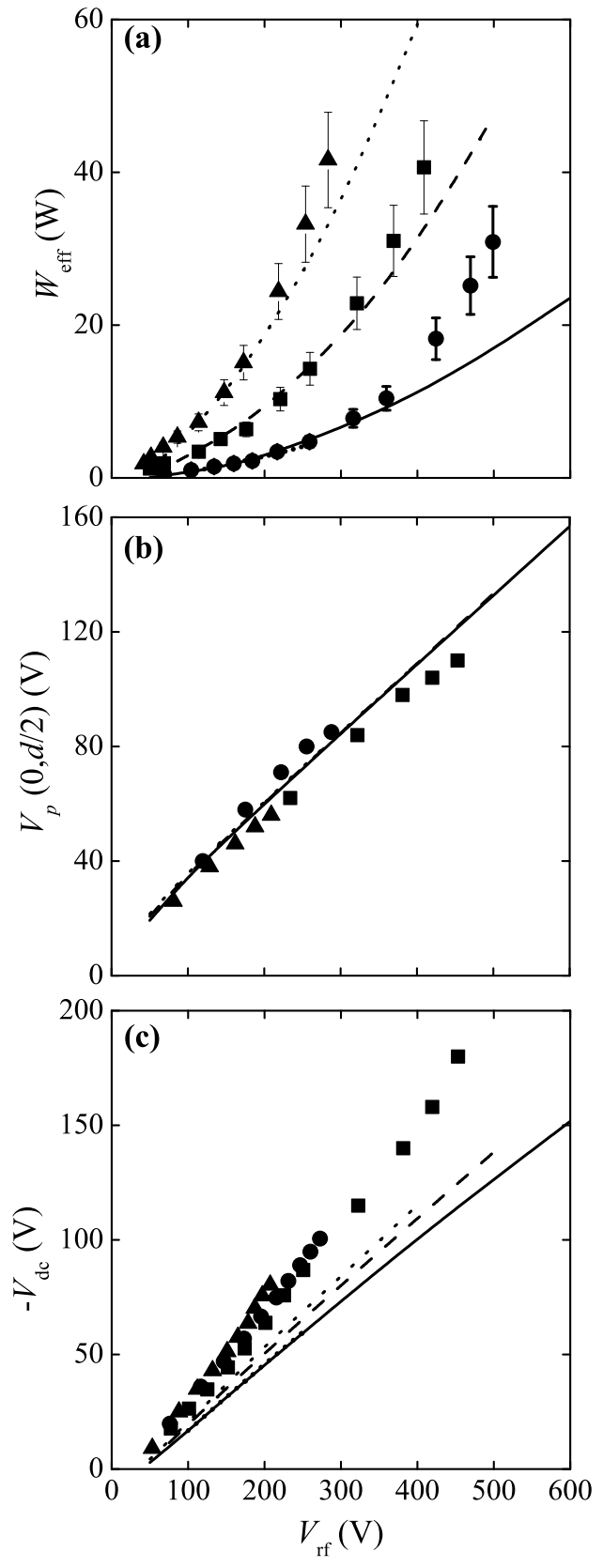

Figure 5. Electrical parameters as a function of $V_{\mathrm{rf}}$ for a $\mathrm{H}_{2}$ discharge at $p=0.3$ Torr. (a) Effective power coupled to the plasma; (b) time-average plasma potential at $r=0$ and $z=d / 2 ;$ (c) self-bias voltage. The curves (simulations) and the points (measurements) were obtained at frequencies $13.56 \mathrm{MHz}$ (solid curves and circles), $27.12 \mathrm{MHz}$ (dashed and squares), and $40.68 \mathrm{MHz}$ (dotted and triangles). The shortdotted curves in (a) and (c) (almost coinciding with the solid curves) were obtained at $13.56 \mathrm{MHz}$ including all corrective terms in the ion and the electron flux equations. 

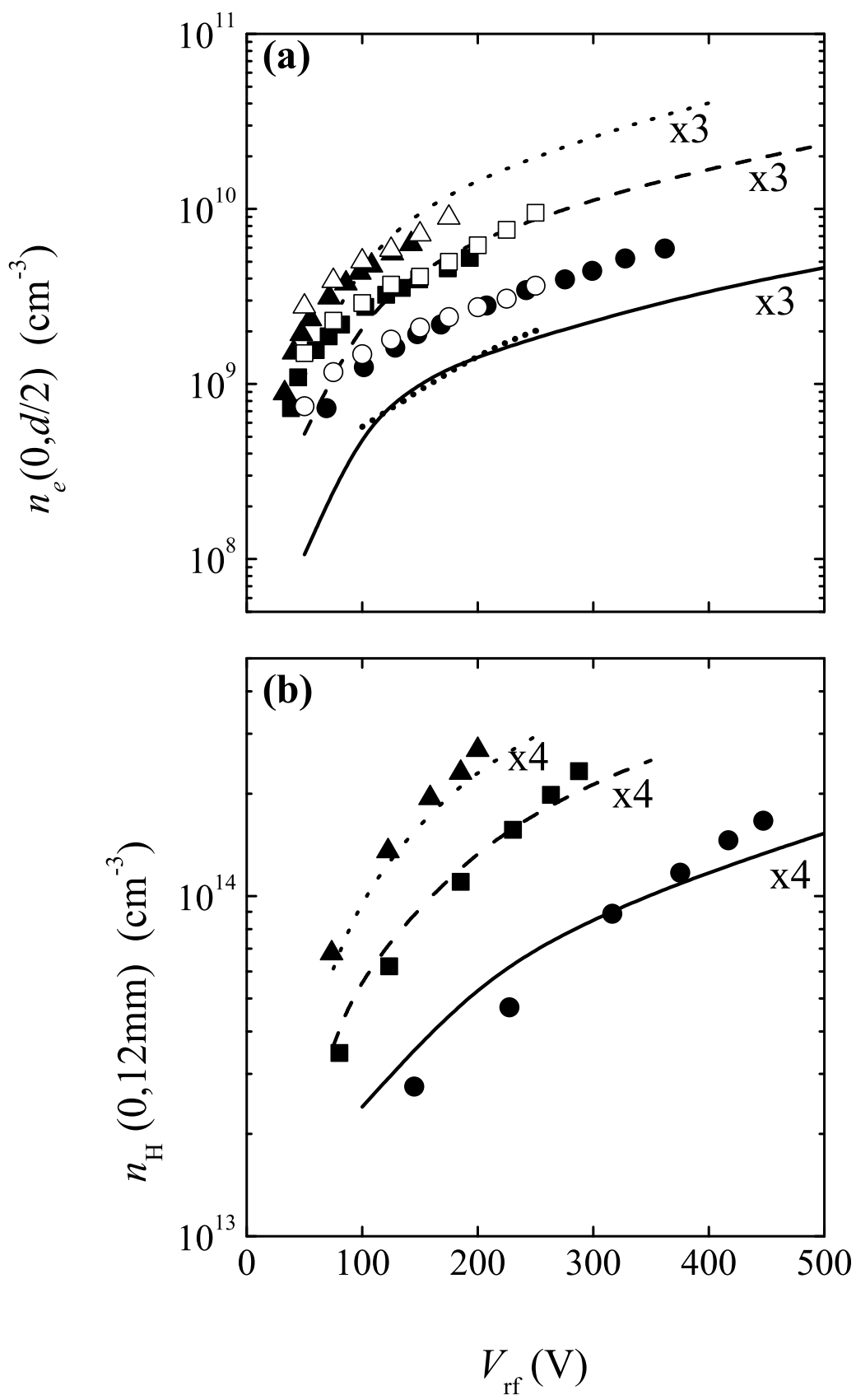

Figure 6. Time-average densities of the electrons (at $r=0$ and $z=d / 2)(\mathbf{a})$ and the $\mathrm{H}$ atom (at $r=0$ and $z=12 \mathrm{~mm}$ ) (b), as a function of $V_{\mathrm{rf}}$, for a $\mathrm{H}_{2}$ discharge at $p=0.3$ Torr. The curves and the points are as in Fig. 5 . The experimental $n_{e}$ values were obtained using a planar probe (closed symbols) or a cylindrical Langmuir probe (open) in (a) and TALIF measurements in (b). Simulations were multiplied by 3 for $n_{e}$ and by 4 for $n_{\mathrm{H}}$. 


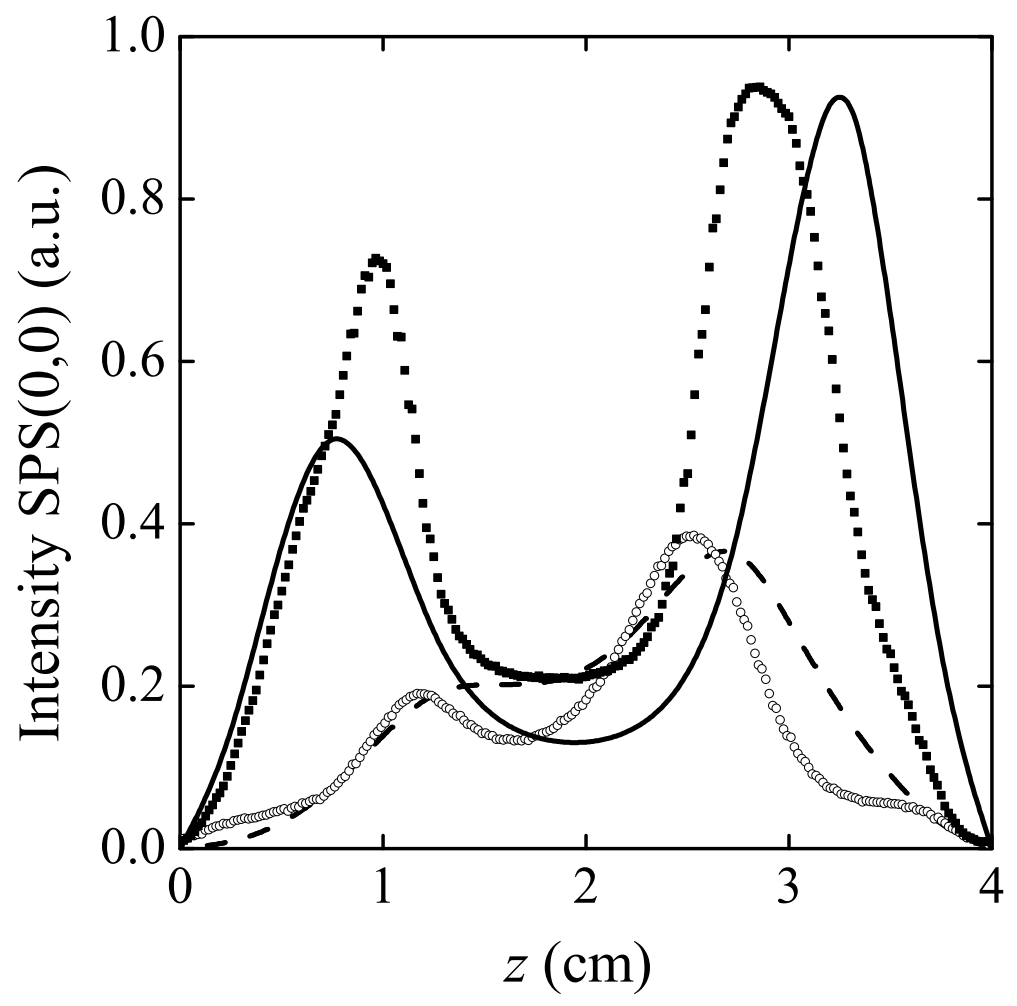

Figure 7. Time- and radial- average radiative intensity of the SPS $(0,0)$ band with a $\mathrm{N}_{2}$ discharge, measured (points) and calculated (lines) between the electrodes for the following work conditions (at $V_{\mathrm{rf}} \simeq 145 \mathrm{~V}$ ): $p=0.75$ Torr and $W_{\text {eff }}=4 \mathrm{~W}$ (solid line and closed squares); $p=0.15$ Torr and $W_{\text {eff }}=16 \mathrm{~W}$ (dashed and open circles). For each condition, the maxima of the experimental and the calculated curves were normalized to the same value. 

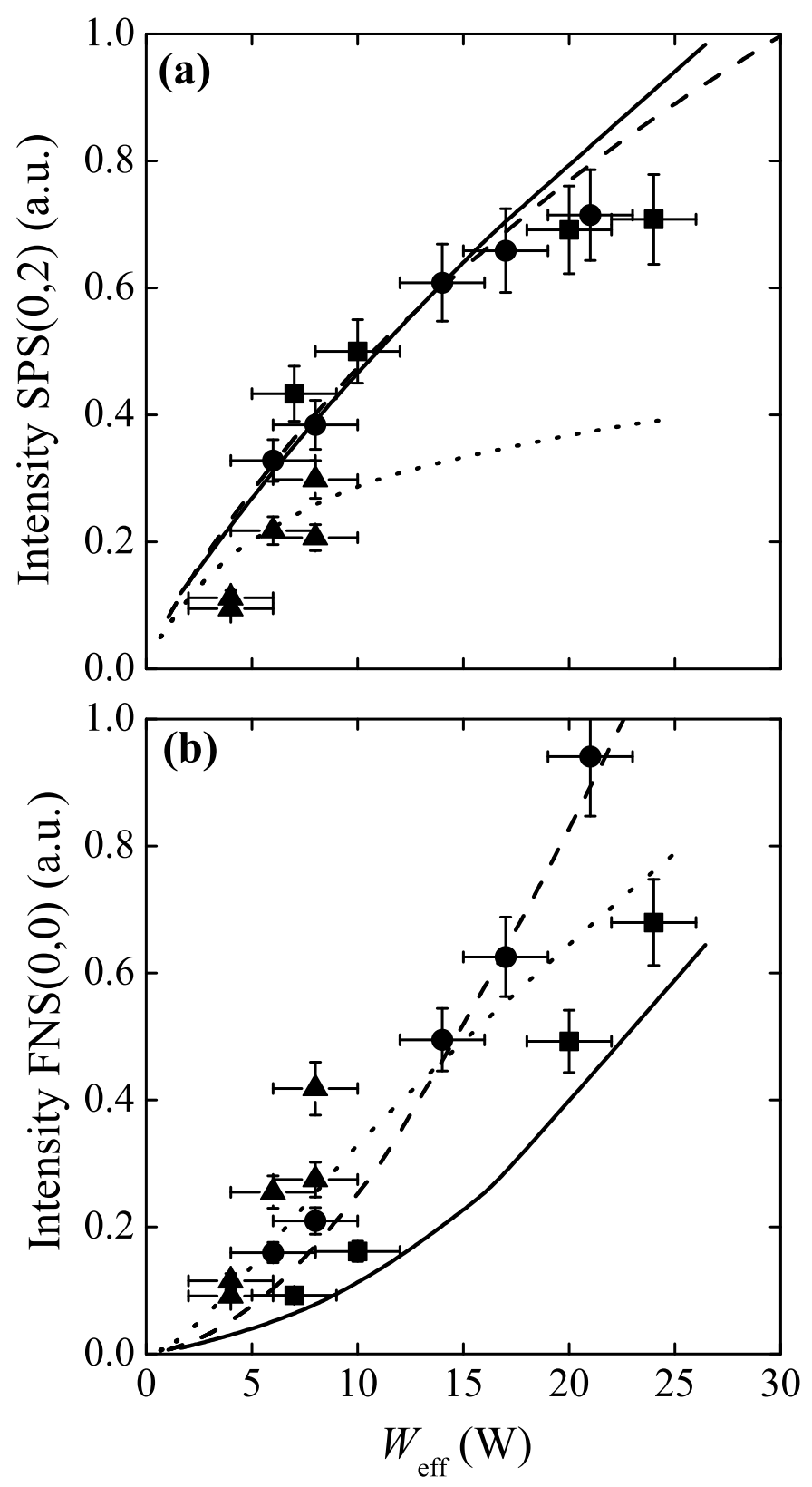

Figure 8. Space-time average radiative intensities, as a function of $W_{\text {eff }}$, of the $\operatorname{SPS}(0,2)$ band (a) and the $\operatorname{FNS}(0,0)$ band (b) with a $\mathrm{N}_{2}$ discharge, calculated (curves) and measured (points) for the following pressures (in Torr): 0.75 (solid line and squares); 0.45 (dashed and circles); 0.15 (dotted and triangles). Within each subfigure all simulation values are normalized to one experimental point obtained at: $\sim 7 \mathrm{~W}$ and 0.45 Torr for the $\operatorname{SPS}(0,2) ; \sim 7 \mathrm{~W}$ and 0.15 Torr for the $\operatorname{FNS}(0,0)$. Simulations were averaged radially, over the entire discharge cross section, and axially, over $1 / 3$ of the interelectrode distance below the driven electrode (corresponding to the region where the OES measurements were carried out). 


\section{Tables and table captions}

Table 1. Extra loss channels for dimmers considered in model update. $p$ is the pressure in Torr.

\begin{tabular}{ll}
\hline Reaction & Rate coefficient $\left(\mathbf{c m}^{3} \mathbf{s}^{-1}\right)$ \\
\hline $\mathrm{SiH}_{4}+\mathrm{Si}_{2} \mathrm{H}_{2} \longrightarrow \mathrm{H}_{2}+\mathrm{Si}_{3} \mathrm{H}_{4}$ & $2 \times 10^{-10}$ \\
$\mathrm{SiH}_{4}+\mathrm{Si}_{2} \mathrm{H}_{3} \longrightarrow \mathrm{H}_{2}+\mathrm{Si}_{3} \mathrm{H}_{5}$ & $2 \times 10^{-10}$ \\
$\mathrm{SiH}_{4}+\mathrm{Si}_{2} \mathrm{H}_{4} \longrightarrow \mathrm{Si}_{3} \mathrm{H}_{8}$ & $3 \times 10^{-10}\left(1-(1+0.5 p)^{-1}\right)$ \\
$\mathrm{H}+\mathrm{Si}_{2} \mathrm{H}_{5} \longrightarrow \mathrm{H}_{2}+\mathrm{Si}_{2} \mathrm{H}_{4}$ & $2 \times 10^{-11}$ \\
$\mathrm{SiH}_{3}+\mathrm{Si}_{2} \mathrm{H}_{5} \longrightarrow \mathrm{SiH}_{4}+\mathrm{Si}_{2} \mathrm{H}_{4}$ & $1.5 \times 10^{-10}$ \\
$\mathrm{Si}_{2} \mathrm{H}_{5}+\mathrm{Si}_{2} \mathrm{H}_{5} \longrightarrow \mathrm{SiH}_{2}+\mathrm{Si}_{3} \mathrm{H}_{8}$ & $1.5 \times 10^{-10}$ \\
\hline
\end{tabular}

Table 2. Loss probabilities of radicals on an a-Si:H covered surface.

\begin{tabular}{llll}
\hline Species & $\zeta$ & $\gamma$ & s \\
\hline electrons, ions & 1 & 1 & 0 \\
$\mathrm{H}$ & 0.7 & 0.7 & 0 \\
$\mathrm{SiH}_{3}, \mathrm{Si}_{2} \mathrm{H}_{5}$ & 0.26 & 0.17 & 0.09 \\
$\mathrm{SiH}_{2}, \mathrm{Si}_{2} \mathrm{H}_{4}$ & 0.6 & 0 & 0.6 \\
$\mathrm{SiH}, \mathrm{Si}_{2} \mathrm{H}_{3}$ & 0.95 & 0 & 0.95 \\
$\mathrm{Si}, \mathrm{Si}_{2} \mathrm{H}_{2}$ & 1 & 0 & 1 \\
\hline
\end{tabular}




\section{References}

[1] Deshmukh S C and Aydil E S 1996 J. Vac. Sci. Technol. B 14738

[2] Viera G, Huet S, Bertran E and Boufendi L 2001 J. Appl. Phys. 904272

[3] Amanatides E, Hammad A, Katsia E and Mataras D 2005 J. Appl. Phys. 97073303

[4] Peng X, Wang Z, Dimitrov D, Boonstra T and Xue S 2007 J. Vac. Sci. Technol. A 251078

[5] Obraztsov A N, Volkov A P, Nagovitsyn K S, Nishimura K, Morisawa K, Nakano Y and Hiraki A 2002 J. Phys. D: Appl. Phys. 35357

[6] Kumar S, Dixit P N, Sarangi D and Bhattacharyya R 2003 J. Appl. Phys. 936361

[7] Denysenko I, Ostrikov K, Cvelbar U, Mozetic M and Azarenkov N A 2008 J. Appl. Phys. 104 073301

[8] Hayashi Y, Krishna K M, Ebisu H, Soga T, Umeno M and Jimbo T 2001 Diamond and Related Materials 101002

[9] Anita V, Butuda T, Maeda T, Takizawa K, Saito N, Takai O 2004 Diamond and Related Materials 131993

[10] Abbas G, Papakonstantinou P, Iyer G R S, Kirkman I W and Chen L C 2007 Phys. Rev. B 75 195429

[11] Hossain M M, Herrmann A S and Hegemann D 2007 Plasma Process. Polym. 4135

[12] Miyajima Y, Henley S J and Silva S R P 2011 Thin Solid Films 5196374

[13] Othman M, Ritikos R, Khanis N H, Rashid N M A, Rahman S A, Gani S M A and Muhamad M R 2011 Thin Solid Films 5194981

[14] Tzeng S-S, Fang Y-L, Chih Y-K, Hu Y-G, Hsu J-S, Wu C-L and Wu G-J 2011 Thin Solid Films $\mathbf{5 1 9} 4870$

[15] Szopa C, Cernogora G, Boufendi L, Correia J J and Coll P 2006 Planet. Space Sci. 54394

[16] Boeuf J P 1988 J. Appl. Phys. 631342

[17] Tsai J H and Wu C 1990 Phys. Rev. A 415626

[18] Hashigushi S 1992 J. Vac. Sci. Technol. A 101339

[19] Dalvie M, Surendra M and Selwyn G S 1993 Appl. Phys. Lett. 623207

[20] Young F F and Wu C H 1993 IEEE Trans. Plasma Sci. PS-21 312

[21] Passchier J D P and Goedheer W J 1993 J. Appl. Phys. 743744

[22] Fiala A, Pitchford L C and Boeuf J P 1994 Phys. Rev. E 495607

[23] Surendra M 1995 Plasma Sources Sci. Technol. 456

[24] Lymberopoulos D P and Economou D J 1995 J. Phys. D: Appl. Phys. 28727

[25] Goedheer W J, Meijer P M, Bezemer J, Passchier J D P and van Sark W G J H M 1995 IEEE Trans. Plasma Sci. PS-23 644

[26] Boeuf J P and Pitchford L C 1995 Phys. Rev. E 511376

[27] Leroy O, Gousset G, Alves L L, Perrin J, Jolly J 1998 Plasma Sources Sci. Technol. 7348

[28] Salabas A, Gousset G and Alves L L 2002 Plasma Sources Sci. Technol. 11448

[29] Salabas A, Marques L, Jolly J, Gousset G and Alves L L 2004 J. Appl. Phys. 954605

[30] Marques L, Jolly J and Alves L L 2007 J. Appl. Phys. 102063305

[31] Chen Z, Rauf S and Collins K 2010 J. Appl. Phys. 108073301

[32] Alves L L, Marques L, Pintassilgo C D, Wattieaux G, Es-sebbar Et, Berndt J, Kovačević E, Carrasco N, Boufendi L and Cernogora G 2012 Plasma Sources Sci. Technol. 21045008

[33] Kim H C, Iza F, Yang S S, Radmilović-Radjenović M and Lee J K 2005 J. Phys. D: Appl. Phys. 38 R283

[34] Kae-Nune P, Perrin J, Guillon J and Jolly J 1994 Jpn. J. Appl. Phys. 334303

[35] Kae-Nune P, Perrin J, Guillon J and Jolly J 1995 Plasma Sources Sci. Technol. 4250

[36] Alves L L 2007 Plasma Sources Sci. Technol. 16557

[37] Davison B and Sykes J B 1958 Neutron Transport Theory (Oxford: Clarendon)

[38] Chantry P J 1987 J. Appl. Phys. 621141

[39] Gallagher A 1986 J. Appl. Phys. 601369 
[40] Kushner M J 1988 J. Appl. Phys. 632532

[41] Sommerer T J and Kushner M J 1992 J. Appl. Phys. 711654

[42] Choi S J, Ventzek P L, Hoekstra R J and Kushner M J 1994 Plasma Sources Sci. Technol. 3418

[43] Perrin J, Leroy O and Bordage M C 1996 Contrib. Plasma Phys. 363

[44] Hertl M and Jolly J 1999 private communication 